\title{
INFLUÊNCIA DA COMPOSIÇÃO DO SUBSTRATO NO DESEMPENHO MORFOFISIOLÓGICO DAS MUDAS DE Trema micrantha (L.) Blume.
}

Influence of substrate composition on the morphophysiological performance of Trema micrantha (L.) Blume seedlings.

Influencia de la composición del sustrato en el rendimiento morfofisiológico de las plántulas de Trema micrantha (L.) Blume.

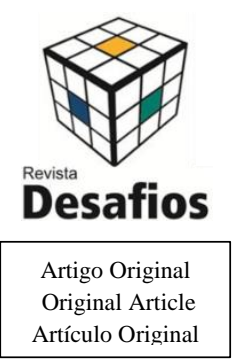

Yaovi Abel Kissi ${ }^{* 1}$, Ivo de Sá Motta ${ }^{2}$, Eny Duboc ${ }^{2}$, Etenaldo Felipe Santiago ${ }^{1,3}$

${ }^{1}$ Grupo de Estudos dos Recursos Vegetais - GERV, Universidade Estadual de Mato Grosso do Sul, Dourados, Brasil

${ }^{2} \operatorname{Dr}$ (a) e Pesquisador (a) Embrapa Agropecuária Oeste (CPAO), Dourados, Brasil.

${ }^{3}$ Dr Docente Universidade Estadual de Mato Grosso do Sul - UEMS, Dourados, Brasil

*Correspondência: kissiabel@yahoo.fr.

Artigo recebido em 11/11/2019 aprovado em 06/11/2020 publicado em 04/03/2021.

\section{RESUMO}

O uso de biossólidos na produção de mudas de espécies nativas é uma alternativa na gestão dos resíduos orgânicos. O sucesso no estabelecimento das mudas no campo depende de vários fatores com destaque para a qualidade do seu meio de crescimento. O presente trabalho teve como objetivo avaliar o desempenho morfofisiológico de mudas de Trema micrantha (L.) Blume produzidas em biossólidos obtidos da vermicompostagem de lodo de esgoto (LE) e capim napier triturado (NT). Foram utilizados os substratos V1=40\%LE $+60 \% \mathrm{NT}$, V2 $=50 \% \mathrm{LE}+50 \% \mathrm{NT}$; V3= $60 \% \mathrm{LE}+40 \% \mathrm{NT}$ e V4 (substrato comercial), considerado tratamento controle. Após 120 dias da semeadura, foram avaliados os dados do crescimento em altura das mudas, de temperatura das folhas, condutância estomática (Gs) e fluorescência da clorofila- $a$. Um maior desempenho morfológico foi observado nas mudas produzidas nos biossólidos, sendo que, as alturas médias foram influenciadas pela composição dos biossólidos. As análises dos dados de condutância estomática, dos parâmetros fenomenológicos da fluorescência da clorofila- $a$ e das curvas do transiente OJIP permitiram detectar possível ocorrência de danos reversíveis no fotossistema II, no caso das mudas produzidas com V2; essas mudanças não foram suficientes para levar o sistema a uma fotoinibição.

Palavras-chave: biossólido, fluorescência da clorofila- $a$, condutância estomática.

\section{ABSTRACT}

The use of biosolids to produce seedlings of native species is an alternative to organic waste management. Success in establishing of seedlings in the field depends on several factors with emphasis on the quality of its growing medium. The objective of the present work was to evaluate the morphophysiological performance of Trema micrantha (L.) Blume seedlings produced with biosolids obtained from vermicomposting of sewage sludge (LE) and crushed napier grass (NT). Then following substrates were used VI=40\% LE +60\% NT, V2=50\% LE + 50\% NT; V3=60\% LE + 40\% NT and V4 (comercial substrate), considered as control treatment. 120 days after sowing, data of seedling height growth, leaf temperature, stomatal conductance $(G s)$ and chlorophyll-a fluorescence were evaluated. A higher morphological performance was observed for seedlings produced using biosolids, whereas mean heights values were influenced by biosolids composition. Analysis of stomatal conductance data, phenomenological parameters of chlorophyll-a fluorescence and OJIP transient curves allowed to detect possible occurrence of reversible damage in photosystem II, in the case of seedlings produced with V2; these changes were not enough to bring the system into a photoinhibition.

Keywords: biosolid, chlorophyll-a fluorescence, stomatal conductance. 


\section{RESUMEN}

El uso de biosólidos en la producción de plántulas de especies vegetales nativas es una alternativa en el manejo de los residuos orgánicos. El éxito del establecimiento de plántulas en campo depende de varios factores con énfasis en la calidad de su medio de cultivo. El presente trabajo tuvo como objetivo evaluar el rendimiento morfofisiológico de plántulas de Trema micrantha (L.) Blume producidas a partir de biosólidos obtenidos de vermicompostaje de lodos de depuradora (LE) y hierba napier (Pennisetum purpureum) triturar (NT). Entonces se usó los sustratos V1= $40 \% L E+60 \% N T, V 2=50 \% L E+50 \% N T ; V 3=60 \% L E+40 \% N T$ y V4 (sustrato comercial), considerado el tratamiento control. Después de 120 días de siembra, se evaluaron los datos de altura de las plántulas, temperatura de la hoja, la conductancia estomática (Gs) y fluorescencia de clorofila-a. Se observó un mayor rendimiento morfológico en plántulas producidas con los biosólidos, las alturas promedio fueron influenciadas por la composición de biosólidos. Los análisis de datos de conductancia estomática, los parámetros fenomenológicos de fluorescencia de clorofila-a y las curvas transitorias OJIP permitieron detectar la posible ocurrencia de daño reversible en el fotosistema II, en caso de plántulas producidas con V2; estos cambios no fueron suficientes para conducir el sistema para fotoinhibición.

Descriptores: biosólido, fluorescencia de clorofila-a, conductancia estomática.

\section{INTRODUÇÃO}

$\mathrm{O}$ rápido crescimento populacional, a urbanização e o moderno estilo de vida são fatores essenciais para o aumento maciço de resíduos sólidos em vários países em desenvolvimento (RAJPAL et al., 2014; SUTHAR, 2009; TCHOBANOGLOUS e KREITH, 2002).

O cultivo de plantas em substratos permite o controle mais rígido da nutrição mineral e da irrigação de forma a proporcionar melhores condições de crescimento para as plantas. Neste tipo de cultivo, também é possível contornar condições desfavoráveis, comumente enfrentadas com o cultivo tradicional em solo, como a baixa fertilidade química, impedimentos físicos, além de problemas de salinização, incidência de pragas e doenças, contaminações adversas, entre outros (GRASSI-FILHO e SANTOS, 2004).

$\mathrm{Na}$ seleção de materiais para uso como componentes ou misturas de substratos, busca-se a obtenção de algumas propriedades, como a relação entre as características de aeração e drenagem, o que permitirá o equilíbrio entre a retenção e a liberação da água e nutrientes.

A adequação nos valores de $\mathrm{pH}$ e salinidade, a otimização de absorção de água e nutrientes pelas raízes são características que colaboram na redução dos custos de transporte, na redução de propágulos de doenças e também para evitar o uso abusivo de defensivos agrícolas (KÄMPF, 2004).

Diversos materiais podem ser utilizados como substratos para o cultivo de espécies vegetais, porém, em algumas situações, pode ser interessante realizar misturas destes para que se possa atingir as melhores condições químicas e físicas para o crescimento das plantas (GRASSI-FILHO e SANTOS, 2004).

A vermicompostagem consiste no uso de minhocas para transformar os resíduos orgânicos em produto humificado denominado vermicomposto (MUNROE, 2007); outro subproduto desse processo é a biomassa de minhoca que pode ser usada como fonte de proteína animal (LALANDER et $a l .$, 2015).

A espécie Trema micrantha (L.) Blume, conhecida popularmente como grandiúva, pertence à família Cannabaceae que, de acordo com Souza e Lorenzi (2005), possui distribuição cosmopolita, incluindo 11 gêneros e cerca de 170 espécies. No Brasil ocorrem dois gêneros e acerca de 15 espécies, sendo que a grandiúva tem ampla distribuição, ocorrendo em 20 estados brasileiros (CARVALHO, 2003). Esta espécie vegetal tem grande versatilidade ecológica: é uma das pioneiras mais utilizadas em reflorestamento heterogêneo, na recuperação e na recomposição de 
áreas degradadas, e sendo também recomendada para restauração de mata ciliar em locais sem inundação, para conservação de solos, na recuperação de áreas erodidas, áreas de mineração e na estabilização de dunas (CARVALHO, 2003).

Variações nas respostas fisiológicas e morfológicas são comuns em plantas de populações naturais, sugerindo um traço adaptativo complexo, evidenciados quando as plantas são submetidas a condições longe do ótimo de desenvolvimento (SANTIAGO e PAOLI, 2007).

Partindo-se da hipótese de que as mudas produzidas em substratos alternativos apresentariam algum tipo de estresse nutricional (por falta ou por excesso de alguns nutrientes) no seu desenvolvimento, esse trabalho teve como objetivo avaliar o desempenho fisiológico das mudas de Trema micrantha produzidas em substratos à base de vermicomposto de lodo de esgoto e capim napier triturado por meio dos dados de temperatura foliar, condutância estomática, fluorescência da clorofila- $a$ e de altura.

\section{MATERIAIS E MÉTODOS}

\section{Origem e beneficiamento das sementes}

Os frutos de Trema micrantha foram coletados de várias árvores localizadas no Distrito Carumbê no estado de Mato Grosso do Sul. Os critérios de seleção das matrizes seguiram as recomendações de Barbosa (2000). O beneficiamento manual dos frutos realizouse no Centro de Estudos em Recursos Naturais (CERNA) da Universidade Estadual de Mato Grosso do Sul com auxílio de peneira. Foram selecionadas as sementes com adequado aspecto morfológico, sem danos aparentes, sendo as mesmas dispostas em bandeja plástica forrada de papel de filtro e submetidas à secagem em condições ambientes durante $48 \mathrm{~h}$.
Decorrido este período, as sementes foram utilizadas sem armazenamento prévio.

\section{Produção de mudas e delineamento experimental}

As mudas foram produzidas usando substratos produzidos na Empresa Brasileira de Pesquisa Agropecuária - Embrapa Agropecuária Oeste de Dourados e um substrato comercial. Os resíduos utilizados na vermicompostagem consistiram na combinação de materiais orgânicos de origem animal e vegetal (lodo de esgoto-LE e capim napier trituradoNT) em diferentes concentrações. Desse modo, as seguintes combinações de resíduos foram usadas na produção das mudas: $\mathrm{V} 1=40 \% \mathrm{LE}+60 \% \mathrm{NT}$, V2= $50 \% \mathrm{LE}+50 \% \mathrm{NT} ; \mathrm{V} 3=60 \% \mathrm{LE}+40 \% \mathrm{NT}$ e V4 o substrato comercial e considerado o tratamento controle.

As mudas foram produzidas na casa de 3 vegetação do CERNA utilizando tubetes de polietileno $55 \mathrm{ml}$ e frequência diária de duas irrigações de 15 minutos cada; o delineamento foi inteiramente casualizado (DIC). Para cada substrato, foram realizadas 4 repetições de 25 sementes sendo um total de 400 sementes utilizadas.

\section{Atributos químicos dos substratos}

As análises químicas dos substratos V1, V2 e V3 foram realizadas no laboratório de solos, resíduos e tecidos vegetais da Embrapa Agropecuária Oeste de Dourados (CPAO) enquanto que os dados químicos do substrato V4 foram fornecidos pela empresa produtora do mesmo (Tabela 1).

Os valores de $\mathrm{pH}$ e de condutividade elétrica (CE) foram determinados pela metodologia proposta pela Embrapa (1997).

A determinação dos macronutrientes foi realizada usando o Espectrômetro de Absorção Ótica 
(chamado também Plasma CPO) modelo Ótima 8300 com uma digestão das amostras baseada na metodologia USEPA 3051, enquanto que para a determinação do teor do $\mathrm{C}$ e $\mathrm{N}$ foi usado o CNHS baseado sobre o método de Dumas.

Tabela 1: Características químicas dos substratos $(\mathrm{V} 1=40 \% \mathrm{LE}+60 \% \mathrm{NT}$, V2 $=50 \% \mathrm{LE}+50 \% \mathrm{NT}$; $\mathrm{V} 3=60 \% \mathrm{LE}+40 \% \mathrm{NT}$ e V4= Substrato comercial; LE- lodo de esgoto, NT-capim napier triturado).

\begin{tabular}{cccccccc}
\hline Substratos & $\mathbf{N}(\%)$ & $\mathbf{C a}^{*}$ & $\mathbf{M g}^{*}$ & $\mathbf{P}^{*}$ & $\mathbf{K}^{*}$ & $\mathbf{p H}$ & $\mathbf{C E}^{*}$ \\
\hline V1 & 1,66 & 6,88 & 0,57 & 4,25 & 0,86 & 4,52 & 1,586 \\
V2 & 1,44 & 8,83 & 0,63 & 4,92 & 0,69 & 4,36 & 2,980 \\
V3 & 1,65 & 8,64 & 0,65 & 4,65 & 0,69 & 4,48 & 2,014 \\
V4 & Traços & 0,009 & 0,003 & Traços & Traços & 5,50 & 0,70 \\
\hline \multicolumn{7}{c}{ Os teores de Ca, Mg, P e K em g/kg, CE em mS.cm ${ }^{-1}}$.
\end{tabular}

\section{Variáveis fisiológicas}

Uma avaliação não destrutiva dos parâmetros fisiológicos foi realizada 120 dias após a semeadura. Assim, as leituras de condutância estomática $(G s)$ foram obtidas com auxílio de um porômetro modelo Delta-T AP4 entre 08 e $10 \mathrm{~h}$ da manhã, escolhendo 4 mudas por repetição; e para cada muda a leitura foi feita a partir das três primeiras folhas situadas no vértice superior.

As medidas da fluorescência da clorofila- $a$ foram realizadas com auxílio de um fluorímetro modelo Fluorpen FP 100-Max a partir das leituras tomadas de 10 mudas (uma folha por muda) por repetição no período compreendido entre 08 e $10 \mathrm{~h}$ da manhã, sendo as folhas mantidas no escuro durante 30 minutos com a utilização de clipes especializados.

Foram analisadas as variáveis instantâneas de fluorescência tais como Fluorescência inicial (Fo), Fluorescência máxima (Fm), Fluorescência variável (Fv), eficiência quântica fotoquímica do fotossistema II (Fv/Fm), bem como os parâmetros de fluxo fenomenológico de energia da fluorescência da clorofila- $a$, da fluorescência transiente polifásica da Clorofila- $a(\mathrm{O}-\mathrm{J}-\mathrm{I}-\mathrm{P})$.

\section{Variável morfológica}

A altura foi a variável morfológica considerada nesse trabalho e medida da base até a gema apical. A leitura foi feita com auxílio de um parquímetro aos 120 dias após a semeadura a partir de 4 mudas escolhidas por repetição.

\section{Análises estatísticas}

Para analisar o desempenho morfofisiológico das mudas, os dados de temperatura foliar, condutância estomática, fluorescência da clorofila- $a$ e os dados de altura foram submetidos a uma análise de variância e as médias foram comparadas pelo teste de Tukey a 5\% de probabilidade. Os testes estatísticos foram realizados utilizando o programa Bioestat 5.3.

\section{RESULTADOS E DISCUSSÃO}

Ainda que os resultados de temperatura foliar sugiram uma tendência de queda dos valores desde $33,37^{\circ} \mathrm{C}$ até o valor mínimo de $30,34{ }^{\circ} \mathrm{C}$ para as mudas produzidas respectivamente nos substratos vermicompostos V1 e V3 e máximo valor de $34,16{ }^{\circ} \mathrm{C}$ para as mudas produzidas com o substrato comercial 
V4 (Tabela 2 e Fig.1), não foi observada diferença

significativa na temperatura das folhas nos substratos

utilizados.

Tabela 2: Valores médios de condutância Estomática (Gs em mmol.m $\left.\mathrm{m}^{2} \cdot \mathrm{s}^{-1}\right)$, altura das mudas (cm) e de temperatura das folhas (Tfoliar em ${ }^{\circ} \mathrm{C}$ ).

\begin{tabular}{cccc} 
Substratos & Gs & Altura & Tfoliar \\
\hline V1= 40\% LE +60\% NT & $130,4 \mathrm{a}$ & $5,10 \mathrm{a}$ & $33,37 \mathrm{a}$ \\
V2= 50\% LE + 50\% NT & $104,6 \mathrm{a}$ & $6,03 \mathrm{a}$ & $32,56 \mathrm{a}$ \\
V3=60\% LE + 40\% NT & $158,9 \mathrm{a}$ & $6,16 \mathrm{a}$ & $30,34 \mathrm{a}$ \\
V4= Substrato comercial & $326,8 \mathrm{~b}$ & $2,93 \mathrm{~b}$ & $34,16 \mathrm{a}$ \\
\hline
\end{tabular}

Para cada variável, as médias em cada coluna seguidas pela mesma letra não diferem estatisticamente entre si pelo teste de Tukey a $5 \%$ de probabilidade.

Figura 1: Medidas de temperatura da superfície das folhas (A) e Box-plot da Condutância Estomática (Gs) em mudas de Trema micrantha produzidas em substratos V1, V2, V3 e V4 (B). Letras iguais não diferiram pelo teste de Tukey a $5 \%$ de probabilidade. $(\mathrm{V} 1=40 \% \mathrm{LE}+60 \% \mathrm{NT}, \mathrm{V} 2=50 \% \mathrm{LE}+50 \% \mathrm{NT}$; V3 $=60 \%$ LE $+40 \%$ NT e V4= Substrato comercial; LE- lodo de esgoto, NT-capim napier triturado).

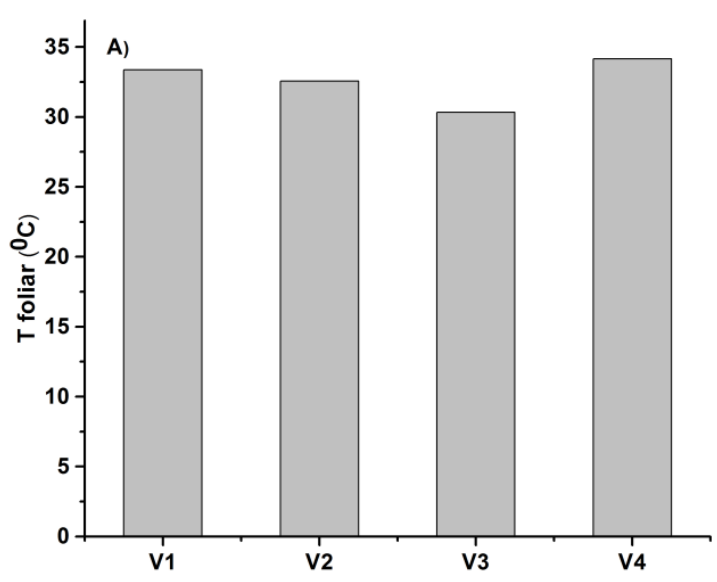

A condutância estomática Gs apresentou diferença significativa entre os valores dos substratos de vermicompostagem de lodo de esgoto (VCL) e napier triturado NT (V1, V2, V3) e do substrato comercial (V4); sendo 104,6 mmol. $\mathrm{m}^{2} \cdot \mathrm{s}^{-1}$ o valor mínimo, observado para o substrato vermicomposto $\mathrm{V} 2$, o valor médio máximo foi de $326,8 \mathrm{mmol} \cdot \mathrm{m}^{2} . \mathrm{s}^{-1} \mathrm{e}$ observado para o substrato comercial V4 (Tabela 2 e Fig.1).

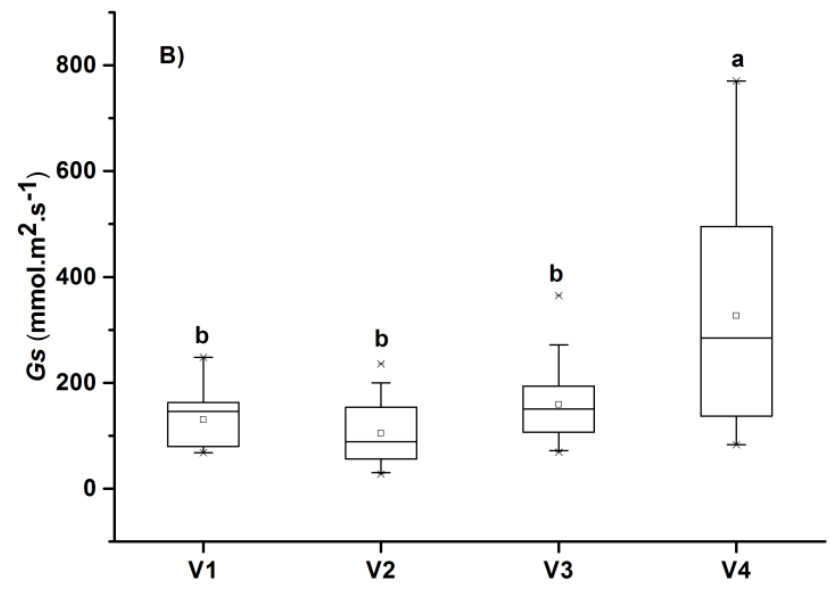

$\mathrm{O}$ crescimento em altura mostrou diferença significativa entre as mudas produzidas nos biossólidos (substratos vermicompostos de lodo de esgoto VCL e napier triturado NT) e as mudas produzidas no substrato comercial. As alturas maiores foram registradas nos substratos VCL e NT, sendo $6,16 \mathrm{~cm}$ a maior altura média registrada no substrato $\mathrm{V} 3$ enquanto que a altura média mais baixa foi de $2,93 \mathrm{~cm} \mathrm{e}$ observada no substrato comercial V4 (Tabela 2). 
O fato do crescimento das mudas não diferir entre os tratamentos V1 a V3 mais serem distintos de V4 podem estar associados aos atributos químicos (tabela 1) que evidenciam diferenças entre os substratos vermicompostados (biossólidos) e o substrato comercial. Entre biossólidos, mesmo a aparente diferença entre os teores de Ca e valores de CE não foram suficientes para interferir no crescimento das mudas. Os dados de crescimento foram similares aos de condutância estomática.

Os fenômenos de abertura e fechamento dos estômatos estão relacionados com a intensidade da luz e o estado hídrico das folhas. A melhor faixa de temperatura para quase todas as plantas é $10-35^{\circ} \mathrm{C}$; a atividade fotossintética das folhas fora desses limites diminui acentuadamente sendo irreversivelmente perdida (TKEMALADZE e MAKHASHVILI, 2016). De acordo com (MORAIS et al., 2007) a radiação elevada combinada com outros fatores ambientais adversos contribui com a diminuição da capacidade fotossintética da planta, podendo levar esta à fotoinibição; por outro lado, no experimento realizado, as condições de radiação foram as mesmas nos diferentes tratamentos, o que nos permite descartar o fator luz para explicar as diferenças de $G s$ e crescimento observados.

Além da radiação, a temperatura também constitui importante fator de influencia no controle estomático. Trema micrantha pertence ao grupo ecológico das pioneiras e as espécies do ambiente tropical toleram temperaturas entorno de $35{ }^{\circ} \mathrm{C}$, sendo considerada a temperatura de $35{ }^{\circ} \mathrm{C}$ ótima para a germinação (PEREIRA et al., 2013). Em um estudo realizado na Floresta Nacional de São Francisco de Paula - RS sobre a relação entre variáveis meteorológicas e o crescimento, Zanon e Finger (2010) verificaram para os indivíduos de Araucaria angustifolia que um aumento da temperatura correspondia ao maior crescimento, cessando-o ou reduzindo-o naqueles períodos com temperaturas mais baixas, ocasiões em que também ocorria um excesso de água no solo.

Em nosso estudo, também se descarta a temperatura como fator relevante para explicar os resultados, posto que não foram observadas diferenças significativas para temperatura foliar (Figura $1 \mathrm{~A}$ ) entre os tratamentos.

Em ambiente tropical, a manutenção de condições adequadas de temperatura foliar é importante para a assimilação uma vez que o complexo supramolecular dos fotossistemas é constituído por componentes protéicos, sendo que diversas reações são catalizadas por enzimas com faixas ótimas de funcionamento que podem variar de acordo com a espécie e/ou ambiente de ocorrência (KHAIRI et al., 6 1976; MONSON et al., 1982; GOLBECK, 2014). Por meio do seu papel na transpiração, os estômatos também ajudam a controlar a temperatura da folha.

Segundo Costa e Marenco (2007), quando observada queda na condutância estomática e consequentemente da fotossíntese ao longo do dia, estes eventos podem ser atribuídos ao aumento do déficit de pressão de vapor (DPV), sem excluir o efeito da alta irradiância. Porém para o presente estudo, as leituras de Gs foram realizadas nas primeiras horas da manhã permitindo reduzir o efeito direto dessas variáveis ambientais no mecanismo de abertura estomática.

O processo de vermicompostagem altera quantitativa e qualitativamente a composição das substâncias húmicas dos materiais orgânicos, favorecendo a formação da matéria orgânica estabilizada, promovendo a mineralização mais lenta e tornando gradual a liberação de nutrientes (CHAQUI et 
al., 2003), este processo também está relacionado ao menor $\mathrm{pH}$ observado nos tratamentos biossólidos quando comparados ao substrato comercial (Tabela 1).

A condutância estomática no caso das plantas dos tratamentos com biossólidos pode ter sofrido influência de ajustes fisiológicos no aparato fotossintético resultado da velocidade moderada da disponibilidade dos nutrientes, sendo o $\mathrm{pH}$ desses substratos mais ácidos (na faixa 4,3 - 4,5) do que o pH do substrato comercial V4 (Tabela 1). Considerando que as mudas foram produzidas no viveiro e com uma frequência de irrigação de duas vezes ao dia, as diferenças entre as proporções de lodo de esgoto e napier triturado podem não ter alterado o estado hídrico das plantas e consequentemente a Gs nos casos de V1, V2 e V3; desta forma, os resultados observados estariam fortemente relacionados aos aspectos químicos dos substratos.

A diferença observada no crescimento em altura entre as mudas produzidas com biossólidos e com o substrato comercial sugere o alto teor de nutrientes presentes nesses substratos devido aos processos de mineralização da matéria orgânica. No caso dos substratos vermicompostos estudados, ainda que o $\mathrm{pH}$ tenha ficado abaixo dos valores ideais para a maioria das plantas comerciais de ambientes mesofíticos (6-6,5) o crescimento 49,14\% superior à condição controle evidencia que os nutrientes presentes foram disponibilizados para as plantas, a despeito do baixo $\mathrm{pH}$. A maior disponibilidade de nutrientes, em especial os maiores teores de $\mathrm{P}$ para as espécies arbóreas tropicais favorecem a maior assimilação do $\mathrm{CO}_{2}$ atmosférico e, por consequência, promovem um maior crescimento e alocação de biomassa (CUNHA et al., 2016). A variação da composição do substrato parece ser um fator determinante no crescimento das mudas produzidas nos biossólidos já que foi observada uma tendência de aumento no crescimento em altura com o aumento da porcentagem de lodo de esgoto na mistura; o crescimento em altura foi também observado por (BACKES et al., 2009) em mudas de Ricinus communis L. submetidas a diferentes proporções de lodo de esgoto. Os elevados valores de condutividade elétrica apresentados pelos substratos à base de LE e NT não foram suficientes para afetar o processo fotossintético das mudas de Trema micrantha como foi observado por (PEÇANHA et al., 2017) em mudas de mamão (Carica papaya L.) cuja fertirrigação resultou em aumento dos valores de condutividade elétrica sendo prejudicial para a fotossíntese e para o crescimento.

Ajustes fisiológicos ocorrem nas plantas no sentido de minimizar efeitos potencialmente danosos às folhas, garantindo, assim, seu funcionamento em condições adversas (YAMASHITA et al., 2000) sendo 7 os parâmetros de fluorescência eficientes na detecção destes ajustes.

Os parâmetros de seção transversal da fluorescência da clorofila- $a$ tais como: a fluorescência mínima no escuro Fo, a Fluorescência máxima Fm, o índice de performance PI; mostraram uma diferença significativa entre um primeiro grupo formado pelos tratamentos V1 e V2 e um segundo grupo formado por V3 e V4, sendo que não houve diferença dentro de cada grupo (Tabela 3).

A eficiência do fotossistema II representada por Fv/Fm foi significativamente diferente entre os três substratos vermicompostos à base de lodo de esgoto $\mathrm{e}$ capim napier triturado V1, V2 e V3; mas as médias da $\mathrm{Fv} / \mathrm{Fm}$ não foram diferentes entre os substratos V3 e V4. A eficiência máxima obtida para V3 e V4 foi de 0,81 e a eficiência mínima obtida foi de 0,69 para V2 (Tabela 3). 
Tabela 3: Parâmetros de seção transversal da fluorescência da clorofila- $a$. Valores médios de Fluorescência mínima no estado adaptado ao escuro (Fo), da fluorescência Máxima (Fm), da Fluorescência Variável (Fv), eficiência quântica fotoquímica do fotossistema II (Fv/Fm) e da Área da Curva OJIP que representa o pool de elétrons transportado para o ciclo de Calvin.

\begin{tabular}{ccccccc}
\hline Substratos & Fo* & Fm* & Fv* & Fv/Fm* & IP* & Área OJIP* \\
\hline V1= 40\% LE +60\% NT & $320,30 \mathrm{~b}$ & $1257,20 \mathrm{~b}$ & $936,90 \mathrm{~b}$ & $0,75 \mathrm{~b}$ & $0,99 \mathrm{~b}$ & $274607,40 \mathrm{~b}$ \\
$\mathrm{~V} 2=50 \% \mathrm{LE}+50 \% \mathrm{NT}$ & $395,50 \mathrm{a}$ & $1312,40 \mathrm{~b}$ & $916,80 \mathrm{~b}$ & $0,69 \mathrm{c}$ & $0,54 \mathrm{~b}$ & $232703,30 \mathrm{~b}$ \\
V3=60\% LE + 40\% NT & $278,00 \mathrm{~b}$ & $1527,60 \mathrm{a}$ & $1249,50 \mathrm{a}$ & $0,81 \mathrm{a}$ & $2,73 \mathrm{a}$ & $309761,90 \mathrm{a}$ \\
V4= Substrato comercial & $294,60 \mathrm{~b}$ & $1550,90 \mathrm{a}$ & $1256,20 \mathrm{a}$ & $0,81 \mathrm{a}$ & $2,35 \mathrm{a}$ & $306139,60 \mathrm{a}$ \\
\hline
\end{tabular}

*Para cada variável, as médias seguidas pela mesma letra na coluna não diferem estatisticamente entre si pelo teste de Tukey a $5 \%$ de probabilidade.

A área da curva OJIP que representa o pool de elétrons transportados para o ciclo de Calvin apresentou uma diferença significativa entre os substratos V2 e V3, sendo que o valor máximo de área foi de 309761,9 para V3 e o mínimo foi de 232703,3 para V2 (Tabela 3).

O transiente OJIP é uma ferramenta de análise da mudança na cinética da fluorescência da clorofila- $a$ que fornece informações detalhadas sobre a estrutura e a função do aparato fotossintético, especialmente do fotossistema II (LAZÁR, 2006). As curvas exibidas são típicas do transiente OJIP da fluorescência da clorofila$a$, as áreas acima da curva de fluorescência entre Fo e Fm foram menores para V1 e V2 indicando que as mudas produzidas nos substratos V3 e V4 tiveram maiores estoques de aceptores de elétrons do PSII devido ao desbalanço entre o fluxo de elétrons do lado doador (Yz ) para o centro de reação (RC) e o fluxo de elétrons do RC para o lado aceptor (QA); mas esse desbalanço não está seguido da diminuição da fluorescência máxima $(\mathrm{Fm})$ como referenciado por Strasser (1997). Observou-se uma tendência de aumento no crescimento em altura das mudas com o aumento da porcentagem de lodo do esgoto na composição dos biossólidos. Do mesmo modo que o substrato comercial V4 na sua composição apresentou nível baixo de potássio e fósforo e não provocando sintomas visíveis de deficiência nutricional nas mudas de Trema micrantha, foi observado um rápido declínio das taxas fotossintéticas por Wykoff et al. (1998) nas culturas de Chlamydomonas reinhardtii (Chlamydomonadaceae) transferidas para meios deficientes em fósforo ou potássio; nessa condição a taxa fotossintética foi reduzida de $76 \%$ em $24 \mathrm{~h}$ e de $96 \%$ em $48 \mathrm{~h}$ sem produzir alterações na viabilidade celular.

Em um estudo da fluorescência da clorofila- $a$ em duas populações de plantas jovens de Cecropia pachystachya Trec. (Urticaceae) aclimatadas e submetidas a elevadas concentrações de $\mathrm{CO}_{2}$, Santiago et al. (2015) estabeleceram que o estado nutricional pudesse afetar muito rapidamente o funcionamento do fotossistema II, sugerindo interferência sobre a atividade enzimática de seus componentes.

Os valores da eficiência do fotossistema II ( Fv/Fm) oscilaram entorno de 0,75 para os substratos de uma maneira geral e de acordo com os valores obtidos por Santos et al. (2014) para plantas de juazeiro (Zizyphus joazeiro Martius) produzidas em condição não estressante. $\mathrm{O}$ leve declino observado na relação 
Fv/Fm no caso do substrato V2 sugere a ocorrência de regulação fotoprotetora reversível do fotossistema II. Os valores $\mathrm{Fv} / \mathrm{Fm}$ combinados com os valores de área da curva OJIP sugerem para os substratos testados um funcionamento do aparato fotossintético sem fotoinibição ao longo do desenvolvimento das mudas.
O fluxo fenomenológico de energia da fluorescência da clorofila- $a$ apresentou diferença entre os valores médios para todos os tratamentos; observouse uma tendência de redução da intensidade dos parâmetros $\mathrm{ABS} / \mathrm{RC}$, TRo/CS e DIo/CS; os substratos V1, V2 e V3 apresentaram diferenças significativas para os parâmetros ABS/RC e DIo/CS (Fig.2).

Figura 2: Parâmetros do fluxo fenomenológico de energia da fluorescência da clorofila- $a$ das mudas de Trema micrantha produzidas com os substratos V1, V2, V3 e V4 (Controle). Para cada parâmetro, letras iguais não diferiram pelo teste de Tukey a 5\% de probabilidade.

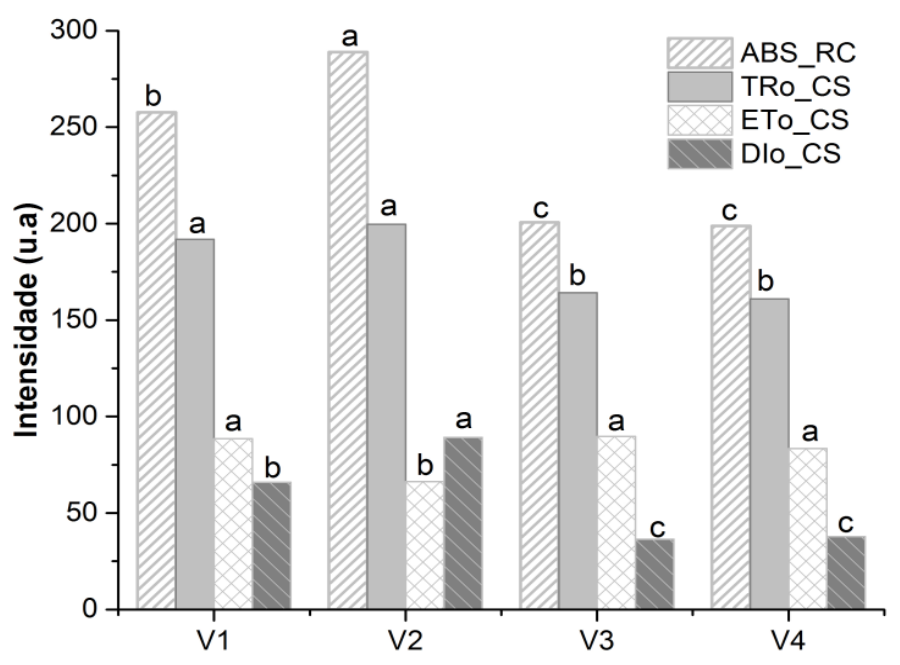

O elevado valor de DIo/Cs apresentado pelas mudas produzidas no substrato V2 indica que grande parte da energia absorvida está sendo dissipada em forma de calor ou de fluorescência, provocando uma redução dos valores de $\mathrm{ETo} / \mathrm{Cs}$ que representa a quantidade de elétrons transportada por seção transversal.

A análise das curvas O-J-I-P mostra as mudanças ocorridas nas diferentes fases. Nos primeiros milissegundos (intervalo O-J), foi observada uma quase superposição das curvas de cinética da fluorescência para os substratos V1, V3 e V4, sendo que a intensidade da fluorescência inicial foi entorno de $300 \mathrm{mmol} . \mathrm{m}^{2} . \mathrm{s}^{-1}$ para esses tratamentos; enquanto que a curva do substrato V2 apresentou uma tendência similar às anteriores com uma intensidade de fluorescência inicial entorno de $400 \mathrm{mmol} \cdot \mathrm{m}^{2} \cdot \mathrm{s}^{-1}$. Na fase J-I (entre 1 e $10 \mathrm{~ms}$ ), observou-se uma ligeira queda na tendência da curva V2 e de uma disjunção das curvas V1, V3 e V4. Na fase I-P (entre 10 e $100 \mathrm{~ms}$ ), observou-se uma queda abrupta seguida de uma subida nas tendências das curvas V1 e V2. Após 100 ms, todas as curvas decresceram de uma maneira geral (Fig.3 e Fig.4).

Figura 3: Alterações da cinética da fluorescência O-J-I-P, observadas nas folhas das mudas de Trema micrantha produzidas com os substratos V1, V2, V3 e V4 (Controle). 


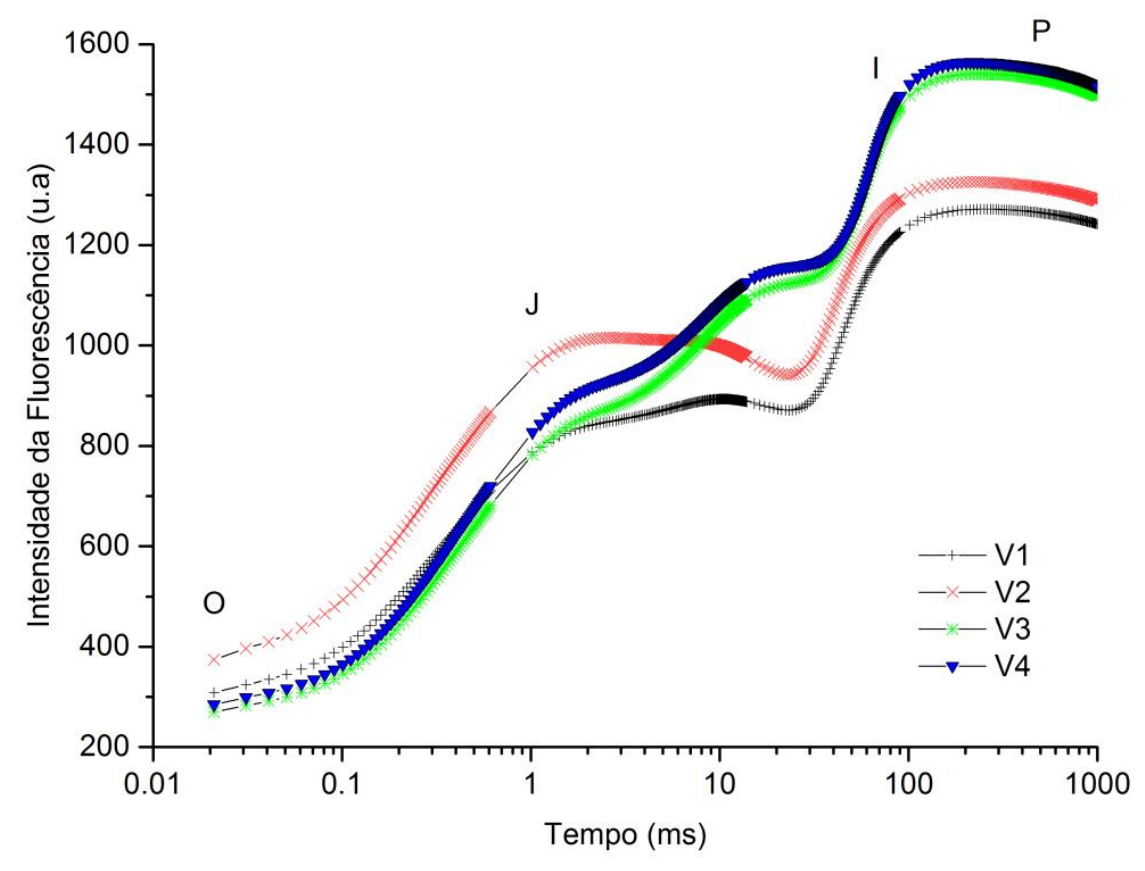

Figura 4: Fluorescência transiente da Clorofila- $a$ (O-J-I-P) de folhas de Trema micrantha produzidas com os substratos V1, V2, V3 e V4 adaptadas ao escuro; análise das etapas O-J, J-I e I-P.
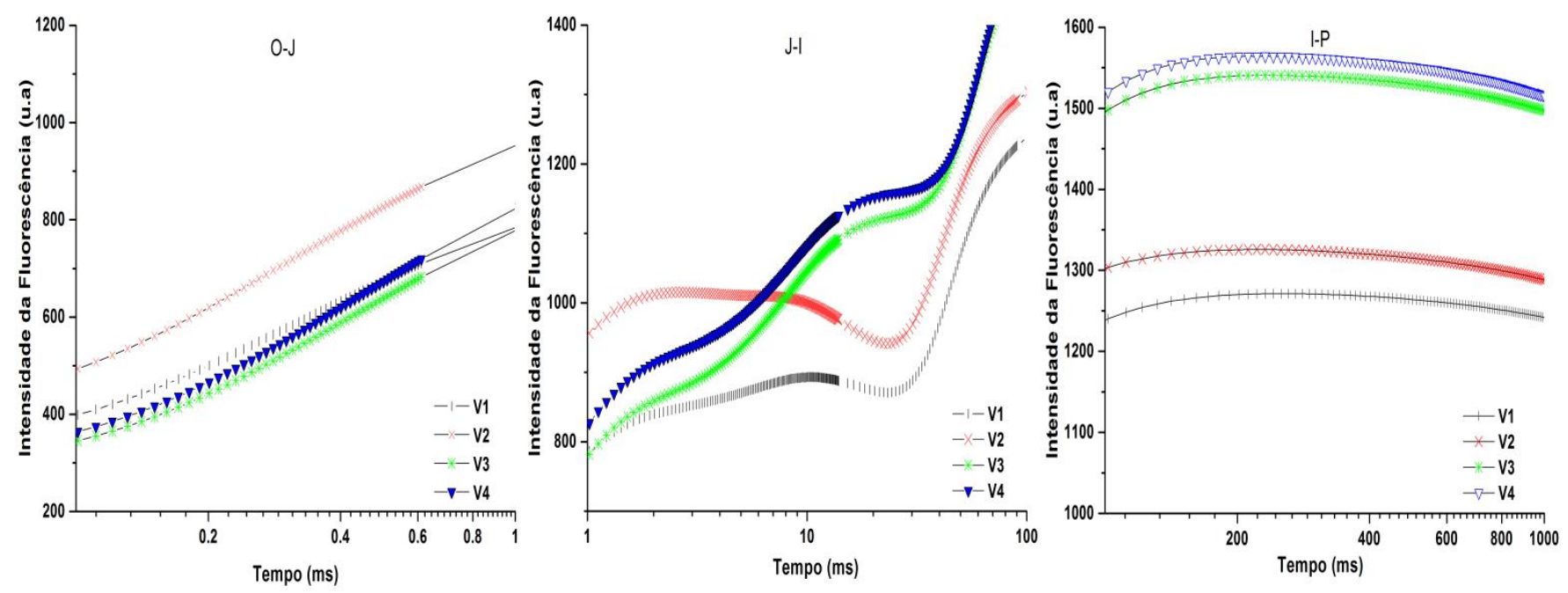

A análise dos espectros de emissão de fluorescência da clorofila- $a$ em diferentes etapas O-J, J-I e I-P mostrou na fase O-J uma quase sobreposição das curvas com exceção da curva do biossólido V2 que apresentou na sua composição mais altos teores de macronutrientes, maior valor de CE e mais baixo valor de $\mathrm{pH}$, reforçando a ideia da maior sensibilidade do aparato fotossintético nestas condições, com menor eficiência quântica fotoquímica e índice de performance (Tabela 3), ainda que não tenha afetado o crescimento das plantas de $T$. micantha. De modo diferente, a inibição do crescimento e do desempenho vegetativo foi observada por Cavalcante et al. (2010) 
em mudas de crisântemo cv. Mirama com o aumento da condutividade elétrica (CE) da solução nutritiva.

A fase O-J ou fase térmica normalmente é afetada por estressores tais como excesso de luz, calor, seca ou inibidores fotossintéticos como a DCMU que atua bloqueando o fluxo de elétrons entre Qa e o aceitador secundário Qb (GOVINDJEE, 2004; SUZUKI et al., 2011). O comportamento dos espectros na fase J-I sugere interferências na transferência dos elétrons entre $\mathrm{Qa}$ e $\mathrm{Qb}$ otimizadas por $\mathrm{CO}_{2}$, isto pode estar relacionado com a indução do íon bicarbonato na proteína D1 e complexo D2 (GOVINDJEE, 2012; SANTIAGO et al., 2015).

Ainda que os parâmetros de crescimento, condutância estomática e temperatura foliar apontem a eficiência dos substratos biossólidos, os parâmetros de emissão de fluorescência da clorofila- $a$ apontam para respostas de sensibilidade das plantas às diferentes proporções.

\section{CONCLUSÃO}

Os substratos vermicompostados, a base de resíduos de lodo de esgoto e capim napier mostram viabilidade na produção de mudas de Trema micrantha.

Quanto aos aspectos químicos, os biossólidos utilizados disponibilizaram nutrientes às plantas cumprindo de modo satisfatório o papel do substrato comercial quando acrescido de nutrientes.

Os parâmetros de emissão de fluorescência da clorofila- $a$ evidenciaram a sensibilidade das mudas de Trema micrantha às diferentes proporções, sugerindo que estudos relacionados à modificação dos parâmetros de pH e CE dos substratos biossólidos são necessários para tornar mais eficiente a disponibilidade dos nutrientes presentes nestes.

\section{AGRADECIMENTOS}

Agradecemos à Universidade Estadual de Mato Grosso do Sul (UEMS) pela oportunidade de matrícula no programa de Pós-Graduação em Recursos Naturais; à Empresa Brasileira de Pesquisa Agropecuária de Dourados (Embrapa-CPAO) pelo apoio logístico. O presente trabalho foi realizado com apoio da Coordenação de Aperfeiçoamento de Pessoal de Nível Superior - Brasil (CAPES) - Código de Financiamento 001; foi também financiado em parte pela Fundação de Apoio ao Desenvolvimento do Ensino, Ciência e Tecnologia do Estado de Mato Grosso do Sul (FUNDECT-MS).

Todos os autores declararam não haver qualquer potencial conflito de interesses referente a este artigo.

\section{REFERÊNCIAS}

BACKES, C.; PAULO, L.C.; MAXIMINO, F.D.; JOSÉ, G.G.L.; APARECIDA, M.K.T.; LYRA, V.B. Efeito do lodo de esgoto e nitrogênio na nutrição e desenvolvimento inicial da mamoneira. Bioscience Journal, Uberlândia, v. 25, n. 1, p. 90-98, 2009.

BARBOSA, L.M. Considerações Gerais e Modelos de Recuperação de Formações Ciliares. In: Rodrigues RR, Leitão-Filho HR. EDUSP. São Paulo: FAPESP., p. 289-312, 2000.

CARVALHO, P.E.R. Informação Tecnológica (Coleção Espécies Arbóreas Brasileiras). Brasília: Embrapa Florestas, v. 1, 2003.

CAVALCANTE, M.Z.B.; PIVETTA, K.F.L.; CAVALCANTE, I.H.L.; CAVALCANTE, L.F.; BELLINGIERI, P.A.; CAMPOS, M.C.C. Electrical conductivity of nutrient solution for chrysanthemum pot cultivation. Revista Brasileira de Ciência do Solo, Viçosa, v. 34, n. 3, p. 747-756, 2010.

CHAQUI, H.I.; ZIBILSKE, L.M.; OHNO, T. Effects of earth-worm casts and compost on soil microbial activity and plant nutrient availability. Soil Biology and Biochemistry. Oxford, v. 35, p. 245-302, 2003. 
COSTA, G.F.; MARENCO, R.A. Fotossíntese, condutância estomática e potencial hídrico foliar em árvores jovens de andiroba (Carapa guianensis). Acta Amazonica, v. 37, n. 2, p. 229-234, 2007.

CUNHA, H.F.V; GONÇALVES, J.F.C; SANTOS JUNIOR, U.M; FERREIRA, M.J; PEIXOTO P.H.P. Biomassa, trocas gasosas e aspectos nutricionais de plantas jovens de pau de balsa (Ochroma pyramidale (Cav. Ex Lamb.) Urb.) submetidas à fertilização fosfatada em ambientes contrastantes de irradiância. Scientia Forestalis, v. 44, p. 215-230, 2016.

GOLBECK, J.H. Van Der Est, A (eds.), The Biophysics of Photosynthesis. Biophysics for the Life Sciences, v. 11, Part V. Springer, New York, p. 433-450, 2014.

GOVINDJEE, A.S. Chlorophyll Fluorescence: A Bit of Basics and History. In: Papageorgiou, G.C.; Govindjee (Eds.), Chlorophyll a fluorescence: a signature of photosynthesis. Springer, Berlin, p. 1-42, 2004.

GOVINDJEE, A.S. Chlorophyll a fluorescence induction: a personal perspective of the thermal phase, the J-I-P rise. Photosynthesis Research, v. 113, p. 15-61, 2012.

GRASSI-FILHO, H.; SANTOS, C.H. Importância da relação entre os fatores hídricos e fisiológicos no desenvolvimento de plantas cultivadas em substratos. In: BARBOSA, J.G.; MARTINEZ, H.E.P.; PEDROSA, M.W.; SEDIYAMA, M.A.N. (Eds.), Nutrição e adubação de plantas cultivadas em substrato. Viçosa: UFV, p.78-91, 2004.

KÄMPF, A. Evolução e perspectivas do crescimento do uso de substratos no Brasil. In: BARBOSA, J.G.; MARTINEZ, H.E.P.; PEDROSA, M.W.; SEDIYAMA, M.A.N. (Eds.), Nutrição e adubação de plantas cultivadas em substrato. Viçosa: UFV, p.0310, 2004.

KHAIRI, M.M.A.; HALL, A.E. Comparative studies of net photosynthesis and transpiration of some citrus species and relativies. Physiologia Plantarum, v. 36, p. 35-39, 1976.

LALANDER, C.H.; KOMAKECH, A.J.; VINNERAS, $B$. Vermicomposting as manure management strategy for urban small-holder animal farmsKampala case study. Waste Management, v. 39, p. 96103, 2015.
LAZÁR, D. The Polyphasic chlorophyll a fluorescence rise measured under high intensity of exciting light. Functional Plant Biology, v. 33, p. 9-30, 2006.

MONSON, R.K.; STIDHAM, M.A; WILLIAMS, G.J; EDWARDS, G.E.; URIBE, E.G. Temperature dependence of photosynthisis in Agropyron smithii Rydb. I. Factors affecting net $\mathrm{CO}_{2}$ uptake in intact leaves and contributionfrom ribulose-1,5biphosphate carboxylase measured in vivo and in vitro. Plant Physiology, v. 69, p. 921-928, 1982.

MORAIS, R.R.; GONÇALVES, J.F.; SANTOSJÚNIOR, U.M.; DUNISCH, O.; SANTOS, A.L.W. Chloroplastid Pigment Contents and Chlorophyll $a$ Fluorescence in Amazonian Tropical Three Species. Revista Árvore, v. 31, n. 5, p. 959-966, 2007.

MUNROE, G. 2007. Manual of On-farm vermicomposting and vermiculture. Organic. Agriculture Centre of Canada. 56p. Disponível em: https://fr.scribd.com/doc/47505555/Manual-of-OnFarm-vermicomposting-and-vermiculture-GlennMunroe. Acesso em: 20/09/2019.

PEÇANHA, A.L.; SILVA, J.R.; RODRIGUES, W.P.;

FERRAZ, T.M.; NETTO, A.T.; LIMA, R.S.N.; LOPES, T.S.; RIBEIRO, M.S.; DEUS, B.C.S.; COUTO, T.R.; SCHAFFER, B.; CAMPOSTRINI, E. Leaf gas exchange and growth of two papaya (Carica papaya L.) genotypes are affected by elevated electrical conductivity of the nutrient solution. Scientia Horticulturae, v. 218, p. 230-239, 2017.

PEREIRA， S.R.; KALIFE， C.; CONTREIRAS RODRIGUES, A.P.D.; LAURA, V.A. Influência da temperatura na germinação de sementes de Peltophorum dubium (Spreng.) Taub. Informativo Abrates, v. 23, n. 3, p. 52-55, 2013.

RAJPAL, A.; ARORA, S.; BHATIA, A.; KUMAR, T.; BHARGAVA, R.; CHOPRA, A.K.; KAZMI, A.A. Cotreatment of organic fraction of municipal solid waste (OFMSW) and sewage by vermireactor. Ecological Engineering, v. 73, p. 154-161, 2014.

SANTIAGO, E.F., PAOLI, A.A.S. Respostas morfológicas em Guibourtia hymenifolia (Moric.) J. Leonard (Fabaceae) e Genipa americana L. (Rubiaceae), submetidas ao estresse por deficiência nutricional e alagamento do substrato. Revista Brasileira de Botânica, v. 30, p. 131-140, 2007. 
SANTIAGO, E.F.; LARENTIS, T.C.; BARBOSA, V.M.; CAIRES, A.R.; MORAIS, G.A; SÚAREZ, Y.R. Can the chlorophyll-a fluorescence be useful in identifying acclimated young plants from two populations of Cecropia pachystachya Trec. (Urticaceae), under elevated $\mathrm{C}_{\mathrm{O} 2}$ concentrations? Journal of Fluorescence, v. 25, p. 49-57, 2015.

SANTOS, J.L.M.; SILVA, E.C; SILVA JUNIOR, C.D. Análise preliminar das trocas gasosas, fluorescência da clorofila-a e potencial hídrico de plantas de juazeiro em uma região semi-árida de Sergipe, Brasil. Scientia Plena, v. 10, 022401, 2014.

SOUZA, V.C.; LORENZI, H. Sistemática: guia ilustrado para a identificação das famílias de Angiospermas da flora brasileira, baseado em APG/II. Nova Odessa: Instituto Plantarum, 2005.

STRASSER, B. Donor side capacity of photosystem II probed by chlorophyll a fluorescence transients. Photosynthesis Research, v. 52, p. 147-155, 1997.

SUTHAR, S. Vermicomposting of vegetable-market solid waste using Eisenia fetida: impact of bulking material on earthworm growth and decomposition rate. Ecological Engineering, v. 35, p. 914-920, 2009.

SUZUKI, K.; OHMORI, Y.; RATEL, E. High Root Temperature Blocks Both Linear and Cyclic
Electron Transport in the Dark During Chilling of the Leaves of Rice Seedlings. Plant Cell Physiology, v. 52, p. 1697-1707, 2011.

TCHOBANOGLOUS, G. KREITH, F. Handbook of Solid Waste Management. McGraw-Hill, USA, 2002.

TKEMALADZE, G.S; MAKHASHVILI, K.A. Climate changes and photosynthesis. Annals of Agrarian Science, v. 14, n. 2, p. 119-126, 2016.

WYKOFF, D.D.; DAVIES, J.P.; MELIS, A.; GROSSMAN, A.R. The Regulation of Photosynthetic Electron Transport during Nutrient Deprivation in Chlamydomonas reinhardtii. Plant Physiology, v. 117, p. 129-139, 1998.

YAMASHITA, N.; ISHIDA, A.; KUSHIMA, H.; TANAKA, N. Acclimation to sudden increase in light favoring an invasive over native trees in subtropical islands, Japan. Oecologia, v. 125, p. 412$419,2000$.

ZANON, M.L.B.; FINGER, C.A.G. Relação de variáveis meteorológicas com o crescimento das árvores de Araucaria angustifolia (Bertol.) Kuntze 13 em povoamentos implantados. Ciência Florestal, v. 20, n. 3, p. 467-476, 2010. 